\title{
ZERO-ENERGY BUILDINGS IN CITIES WITH DIFFERENT CLIMATES AND URBAN DENSITIES: ENERGY DEMAND, RENEWABLE ENERGY HARVEST ON-SITE AND OFF-SITE AND TOTAL LAND USE FOR DIFFERENT RENEWABLE TECHNOLOGIES
}

\author{
UDO DIETRICH \\ REAP Research Group (Resource Efficiency in Architecture and Planning), \\ HafenCity University Hamburg, Germany.
}

\begin{abstract}
Zero-energy buildings (ZEBs) have no fossil energy consumption; this is achieved by optimizing the building and balancing the remaining energy needs by renewables. If this energy can be harvested onsite, on the building's envelope and its estate, a net-ZEB is reached. If supplementary renewable energy has to be produced off-site on compensating land, the ZEB can be reached with such compensating measures (ZEB_CM). Climate and urban density determine how far a ZEB is possible. Temperatures out of comfort range, lack of daylight and overheating by solar radiation may cause energy demand while high insolation or wind speed delivers good preconditions to produce renewable energy on less land. A high urban density avoids urban sprawl and saves land outside of the cities that can be used for other purposes (agriculture and energy production, among others). But, at a certain density, net-ZEB cannot be realized furthermore, and compensating land is necessary. The paper investigates these effects for 15 selected cities around the globe, covering all main climatic conditions. Based on design rules out of literature and own experiences, a prototypical optimized building is derived for each location, and its energy demand is simulated. Standard assumptions for the efficiency of renewable energy systems are used to determine the need of land to cover it. For different urban densities, it can be concluded how far net-ZEB is possible; if necessary, the need for compensating land is calculated. The results show that for cities with moderate climates, the total land use (city plus compensating land) can decrease with increasing urban density if the technology used off-site has high efficiency (like PV). On the other hand, the total land use may increase remarkably with increasing urban density if the used technology off-site has a low efficiency (like the wind for electricity and especially wood pellets for heating). The final understanding is that cities should meet the energy needs on-site by optimized buildings and structures plus renewable energy production (PV on the building's roofs, geothermal systems, etc.).
\end{abstract}

Keywords: compensating measures, different climates, optimized buildings, urban density, zero-energy building.

\section{INTRODUCTION}

Buildings account for a considerable share of energy for their operation and maintenance leading to a significant impact on the environment. It is estimated that $30 \%$ of the global share of energy is consumed by commercial and residential buildings leading to $28 \%$ of global emissions (excluding construction industry) [1]. The demand for energy by buildings is expected to show an upward trend in the coming years. Residential and commercial buildings consume approximately $60 \%$ of the world's electricity [2]. Eighty-two percent of final energy consumption in buildings was supplied by fossil fuels in 2015 (including primary energy input for power generation; traditional use of biomass excluded) [3].

The Paris Agreement on climate change in the year 2015 charted a new course in an effort to check global warming. Sustainable measures towards energy-efficient and low-carbon solutions for buildings and construction can help achieve the central aim of this agreement, a carbon-free society in a few decades. 
The concept of zero-energy buildings (ZEBs) is required to reduce energy consumption and bring down $\mathrm{CO}_{2}$ emission. The 'Zero' refers to the primary (=fossil) energy demand for the services that are necessary to guarantee the user's comfort inside:

- Heating

- Cooling

- Electricity for artificial light

- Electricity for mechanical ventilation (fans)

- Domestic hot water

That target is achieved by increasing the efficiency of the building and balance the energy needs by renewables that are produced either on-site or off-site.

Following the definition, it must be noted that users of a ZEB do not, by far, live carbon-neutral! The electricity for the use itself ('tenants' electricity': PC, server, gadgets, $\mathrm{TV}$ ), the energy for transportation, nutrition, etc. are NOT included and may not be considered negligible.

ZEBs can be divided into two classes: a) net ZEBs and b) ZEBs with compensating measures (ZEB_CM). The difference arises from the location of renewable energy production. In case of net ZEBs, the energy demand is covered on-site, with systems on the building's envelope and/or on the ground of the own estate. In case of a ZEB_CM that energy production on-site is not sufficient to cover the demand, thus supplementary compensating land/alternative renewable energy sources outside of the own estate must be used.

For a net ZEB, it must be stated that there is a competition between the area of usage that has an energy demand and the size of the building's envelope and estate to produce energy to cover it. With an increasing number of storeys, it becomes more and more difficult to balance the demand (the roof area of the building is the main area for renewable energy systems, it does not increase with the increasing number of storeys, the estate remains the same, etc.). Thus, it can be expected that a net ZEB is only possible to a certain, limited number of storeys and urban density.

In the other case, when compensating measures are necessary, a ZEB_CM will use renewable technologies like PV modules, wind turbines or wood pellets for energy generation on the compensating land.

The energy demand of a building is mainly influenced by its location and design; the main aspects are climatic conditions, urban density, orientation and positioning of the building, number of storeys, construction mass, window to wall ratio, daylight access, natural ventilation strategy, shading system, air tightness, thermal insulation, etc.

A second decisive question is whether the building's users have the possibility to use the building adaptively by personally adaptable thermostats, operable windows, shading systems, light switches, etc. and if they have the chance to adapt themselves to different indoor temperatures with their clothing (no dress code).

This paper is based on the hypothesis that the better the building is climate and user adaptive, the lesser is its energy demand.

\section{SCOPE OF WORK/AIM OF THE PAPER}

In this study, 15 major cities around the globe having different climatic characteristics are chosen. Cities exhibiting similar climatic features are grouped to find similarities and differences. Reykjavik, Oslo and Hamburg are in colder locations, having temperatures less than $10^{\circ} \mathrm{C}$ 
for most of the months and demand heating. Chicago and Beijing have both cold and warm months and require both heating and cooling. Cairo and Delhi have a hot and dry climate with the need for cooling. Singapore, Dar es Salaam, Jakarta and Santo Domingo are hot and humid and need air-conditioning almost throughout the year. Sydney, Santiago, Mexico-City and Addis Ababa belong to a widely comfortable category.

For these cities, the following research questions are investigated:

- What is the maximum number of storeys, size of the estate and urban density for which it is possible to reach net ZEB?

- What is the need of land for compensating measures for different urban densities for ZEB_CM? What is the influence of different renewable energy technologies (like PV versus wind turbines, etc.)?

\section{METHODOLOGY}

This research sums up and generalizes findings based on a university's master course. Different groups of international students worked on the different cities having different climates to find the suitability of ZEBs in these cities. Both net ZEBs and ZEB_CM were analysed and design strategies were adopted.

To facilitate the task, the study is carried out with office buildings that are composed of standard office rooms. They have standard conditions in use and design that are easy to describe. Of course, a major portion of the built-up area comprises residential buildings; it also includes retails, schools, hospitals and industries. Residential buildings need less energy than office buildings; thus if the ZEB for office buildings could be achieved, then it could also be achieved for residences.

Furthermore, real urban situations were not regarded since this is too complicated. A quarter of (identical) office building is assumed, well reflecting the effect of reduced daylight access and increased electricity demand for artificial light if buildings are near together and shade each other. It was mentioned that such mono-use quarters are not sustainable. As the scope of the work is to obtain general tendencies and comparable results with similar assumptions for different cities with diverse climatic conditions, it is difficult to consider the real urban situation. Urban density is expressed as the plot ratio, which is the ratio between the area of usage and the area of estate.

\subsection{Standard office room size and equipment}

The standard office room is a unit of $168 \mathrm{~m}^{2}$ with a depth of $14 \mathrm{~m}$ and a width of $12 \mathrm{~m}$, supposed to provide working space for 12 people. This model size could be replicated throughout the whole width and height of the buildings (with supplementing stairs and elevators etc.). Each storey height/floor height in the building is $3.2 \mathrm{~m}$. The time of usage of the building is 11 hours and 5 days a week which is from morning 7 am to evening $6 \mathrm{pm}$ from Monday to Friday.

\subsection{Renewable energy production}

\subsubsection{On-site}

The thermal energy is obtained by a geothermal system (see section 3.2.3) that fills in maximum the whole estate. The coefficients of performance (COP) for cooling and heating 
are 2.5 and 3.5, respectively. The size of the system determines the possible maximal power of the heating or cooling system.

The electricity necessary for heat pumps, artificial lighting and ventilation is received by harvesting solar energy through PV panels. Polycrystalline PV placed flat on the roof is used for the analysis. It is assumed that a surplus can be delivered to the grid and a supplementary need could feed out of the grid, thus the area of the PV modules is determining the annual contribution to the electricity demand of the building.

For a net ZEB, the described renewable energy systems can deliver the whole energy demand. It is determined up to which number of storeys of the building and up to which building distance/urban density that is possible.

\subsubsection{On compensating land}

In case of the assumed number of storeys or the urban densities being higher than the threshold for a net ZEB, a lack of heat and/or electricity is caused. To balance it, compensating land is needed to accomplish the energy demand of the building. Compensating electricity demand is obtained from onshore or offshore wind turbines or PV modules and supplied to the office building.

Transporting thermal energy (heat) from its source of generation to the place of intended use is not preferred as it leads to a lot of energy losses during its transport. Hence, transport of raw material on-site to burn there for heat energy is more economical. Wood pellets are chosen as a compensating measure for thermal energy demand.

Therefore, the area of compensating land depends on the annual energy demand as well as on the chosen type of renewable technology.

\subsubsection{The energy density of different renewable energy systems}

Solar radiation delivers - depending on the location - a few hundred to more than 2000 $\mathrm{kWh} / \mathrm{y}$ energy to a square meter of earth's surface. Renewable energy systems transfer a part of it into usable energy (electricity, heat, material to burn). Figure 1 illustrates that the efficiency of this process differs remarkably from system to system (values based on [4]). Geothermal systems have highest efficiency (with distance) for heating and cooling and PV modules for electricity. Wind turbines onshore or offshore occupy a greater amount of land. But it must be noted that in some cases, it is difficult to have any other use (like agriculture) apart from PV modules in the land that is covered with PV as the land under PVs is dark and dry. Agri-PV is an option but, in any case, there are two systems, plants and PV, in competition for solar energy. The land in between the wind turbines in a wind farm could be used effectively for other purposes.

Energy plants hold the lowest energy density. Energy plants create competition to agriculture for food production and it should be avoided. Wood pellets are a by-product of renewable forestry; besides the limited production, there is no negative impact.

These values give the yearly harvest of renewable energy. The solar offer of $953 \mathrm{kWh} / \mathrm{m}^{2}$ $\mathrm{y}$ and the harvest of PV modules (efficiency about 15\%) refer to the location of Hamburg, Germany. For all other cities under investigation in this article, these values are adapted to the corresponding solar offer at the chosen location.

Data for wind refer to an average wind velocity of $3 \mathrm{~m} / \mathrm{s}$ on land and $5 \mathrm{~m} / \mathrm{s}$ on the sea and are assumed the same for all locations.

Own estimations show that the harvest of geothermal systems varies only slightly with the temperature in the ground and thus the location. The system is assumed as $100 \mathrm{~m}$ deep vertical 


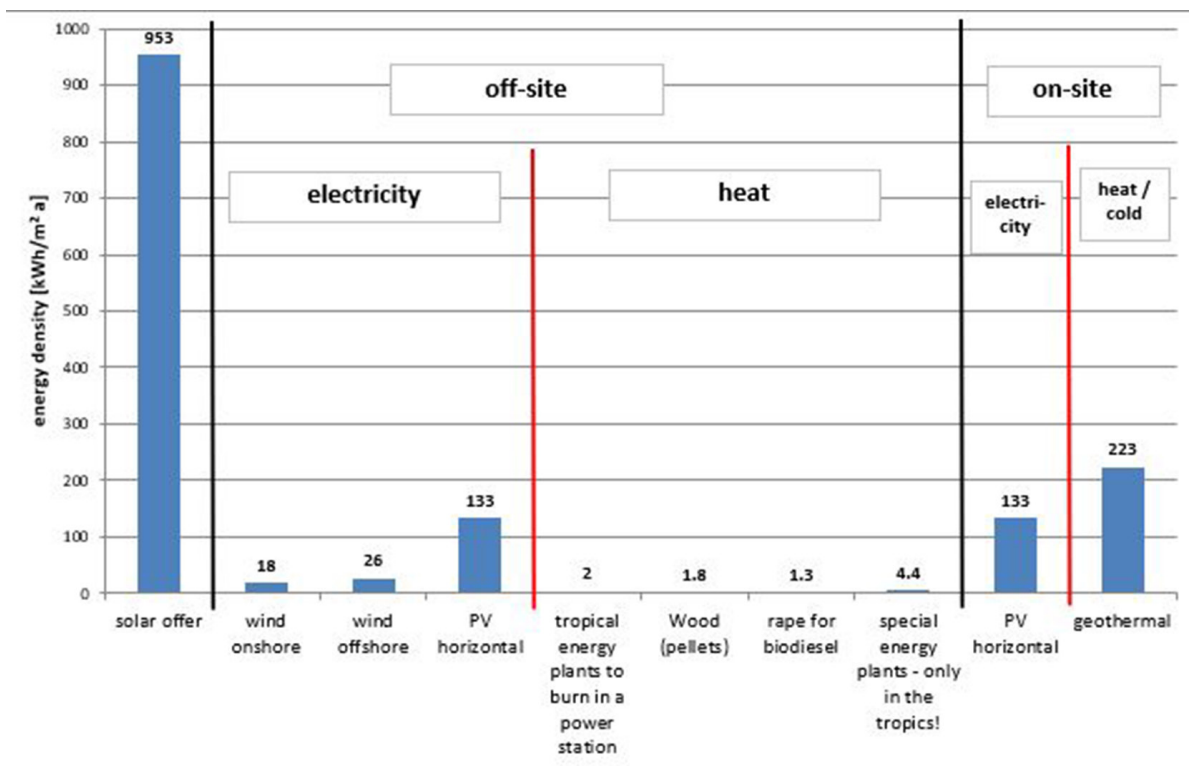

Figure 1: Energy density of different renewable energy systems. Data for solar offer and resulting harvest of PV refer to Hamburg, Germany. The value for geothermal is just to show the dimension for comparison.

borehole heat exchangers with a distance of $7 \mathrm{~m}$. With the assumptions that $1 \mathrm{~m}$ of heat exchanger can deliver $600 \mathrm{Wh} / \mathrm{d}$ thermal energy and that the system is running 6 months a year (heating or cooling season), an energy density of $223 \mathrm{kWh} / \mathrm{m}^{2} \mathrm{y}$ can be derived. This value is only calculated to have a rough impression of the potential of geothermal systems in comparison to the other systems that are included in Fig. 1. Of course, the real potential of the geothermal system will be different for different locations. It is calculated on a daily basis comparing the daily heating/cooling demand and the maximal capacity of the geothermal system.

\subsection{The international style room}

In a first step, the standard office room was assumed as realized in common architecture, as an 'international style' room:

- N-S orientation

- Fully glazed and sealed facades with double heat protection glazing

- Internal shading system

- Air-conditioning $\left(26^{\circ} \mathrm{C}\right)$, mechanical ventilation and artificial light during the whole time of usage

Its energy demand for all the 15 locations was simulated with Primero-Comfort [5], a transient simulation software. Results show that for all locations, more or less, it is not possible to reach a net ZEB with a satisfying urban density; if such a density is assumed, the resulting need for compensating land would be immense. That delivered conviction and motivation that the room should be optimized and adapted to the climate to reduce its energy hunger and to improve the chances to reach a net ZEB or a ZEB_CM with satisfying conditions. 
3.4 Optimization of the standard office room to a climate adaptive one

To find the way to an optimized and adaptive building, several sources were used:

- Climate consultant software [6] is used to understand the temperatures, sun shading, sky cover ratio, wind velocity and humidity.

- Design strategies were derived based on the rules given by the course supervisors and those from the climate consultant.

- Vernacular architecture and best practice examples.

- Also, the opinions and suggestions of the students from these locations were considered.

Firstly, based on the climatic conditions, it was to be decided which month of a year the building can run in which of the following modes:

- Adaptive: Indoor comfort can be maintained with only natural ventilation and heating.

It is assumed that a building can be run adaptively if the monthly mean values of outdoor temperature lie between 10 and $23^{\circ} \mathrm{C}$.

Cities like Addis Ababa, Mexico-City, Sydney and Santiago have a high potential to be run the whole year adaptive.

- Air-conditioned: Indoor comfort can be maintained only with mechanical ventilation and cooling.

It is assumed that a building can be run only air-conditioned if the monthly mean values of the outdoor temperature is above $23^{\circ} \mathrm{C}$ to avoid indoor temperatures out of the comfort range.

Singapore which has a hot and humid climate with an average yearly temperature of $27^{\circ} \mathrm{C}$ uses air-conditioning for 12 months of the year. Santo Domingo, Jakarta and Dar Es Salaam also need air-conditioning nearly throughout the year.

- Heating: Indoor comfort can be maintained only with heating and mechanical ventilation with heat recovery to reduce energy demand.

It is assumed that a building can be run only in this mode if the monthly mean values of outdoor temperature lie below $10^{\circ} \mathrm{C}$.

Reykjavik, Oslo, Chicago, Beijing and partly Hamburg have such strong winter periods. But there is no location where that mode is necessary for the whole year.

- Hybrid: Indoor comfort can be maintained seasonal by running the building adaptively, with air-conditioning or heating.

Buildings could adapt to the surroundings for a few months and depend on cooling or heating and mechanical ventilation for the rest. Chicago is the best example; it can be adaptive for 6 months and needs mechanical ventilation/heating for the rest of 6 months of the year. Hamburg and Beijing also lie in the category of hybrid.

Finally, the architecture could be adapted to the chosen modes (Fig. 2):

- Adjustment (reduction) of room depth for better natural cross ventilation (if of advantage - locations with weak wind velocities) while retaining the area of usage of $168 \mathrm{~m}^{2}$ (an increase of room width).

- Reduction of the window to wall ratio in a way that overheating protection and daylight access is in good symbiosis. The ratio for locations with a dominant cloudy sky is about $50 \%$, and for a dominant clear sky, it is $35 \%$. 


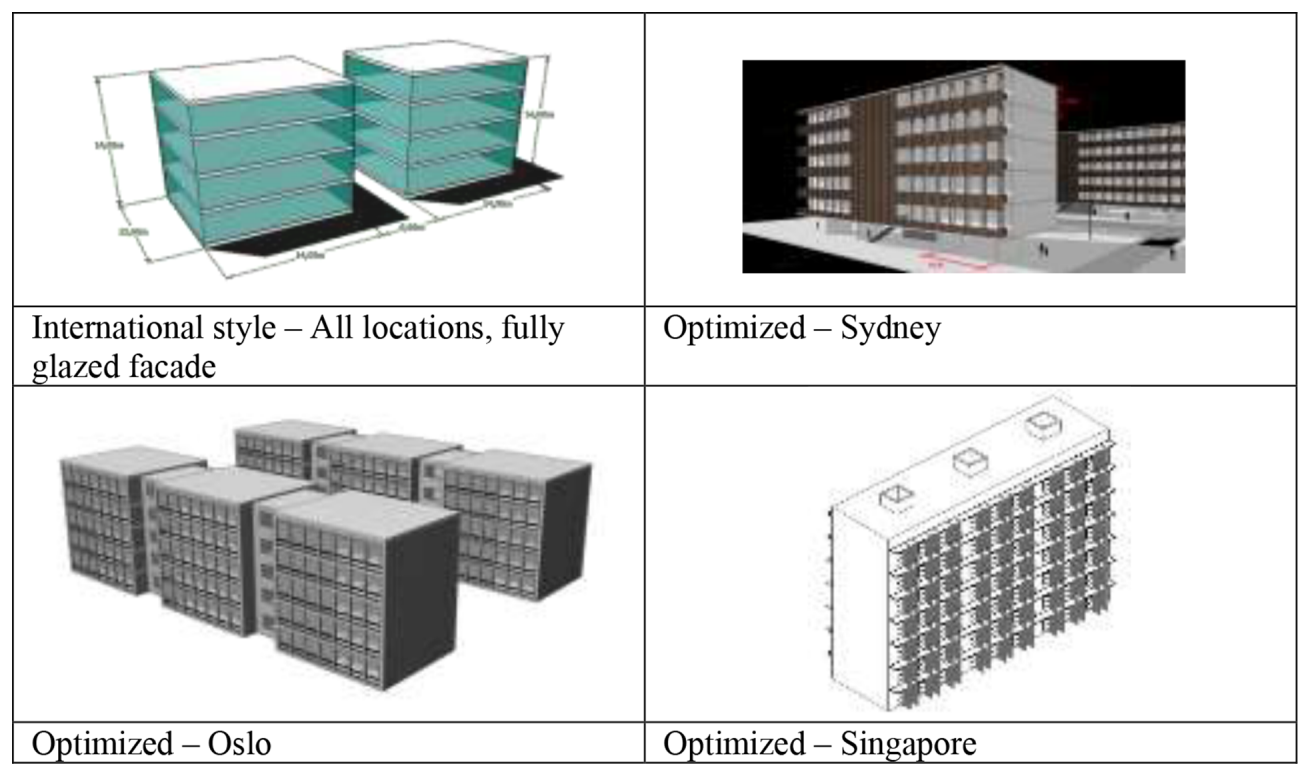

Figure 2: Examples of international style (to find in any city) versus optimized and adaptive buildings for different locations and climates (students work).

- Convenient (external) shading system, glazing and thermal insulation.

- Intelligent size and placement of (operable) windows for daylight and natural ventilation.

- Natural ventilation strategy, especially for night cooling.

- Artificial light can be switched off if daylight is sufficient (500 lx).

These rooms were presented at the end of the university course and then further developed by the author to guarantee that they are optimized for all locations at a corresponding level. Finally, the energy demand for these rooms was simulated with the same software.

\section{RESULTS}

\section{1 $\mathrm{CO}_{2}$ reduction potential of optimized buildings}

Figure 3 gives an overview of the possible reduction potential in primary energy (primary energy factor for heating 1.1, for electricity 3.0) between an international style building and a building that is optimized and adaptive. It can be seen that the potential (and thus the reduction of $\mathrm{CO}_{2}$ emission!) is enormous.

It is also evident from Fig. 3 that the reduction potential is different for different locations. If cooling demand is caused by solar heat gains, it can be reduced (or brought to zero, Hamburg, Oslo, Sydney) by architectural means. If it is caused by temperatures above the comfort range, architectural means can hardly help, and cooling is necessary (Jakarta, Delhi, etc.). If the location is far from the equator, there are many hours of usage where it is dark outside - the demand for artificial light cannot be reduced (Reykjavik, Oslo, etc.). 


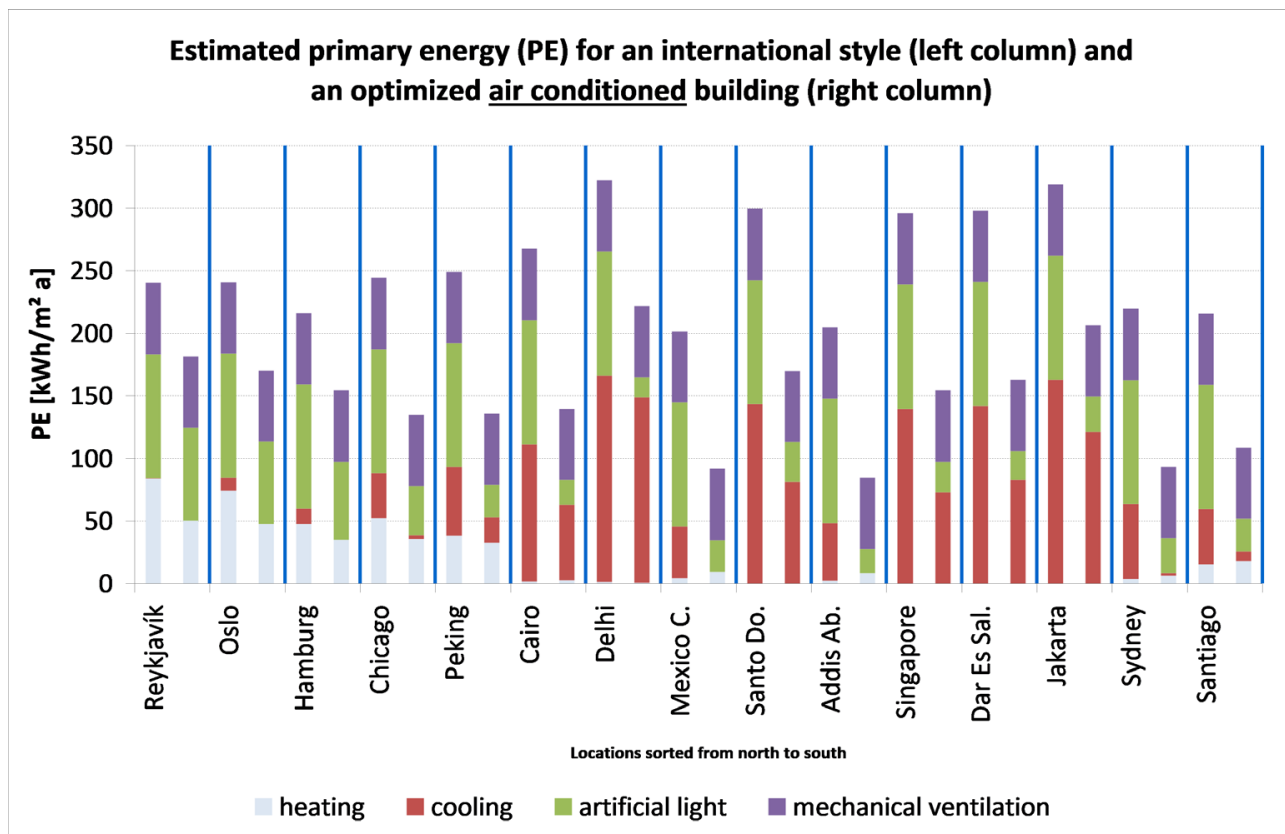

Figure 3: Comparison of the primary energy demand for international style and optimized building.

To deliver a fair comparison, it is assumed that the optimized building has mechanical ventilation and is cooled too (if necessary). Here is further optimization potential; buildings can often be run in adaptive mode (see section 3.4) and without mechanical ventilation.

\subsection{Maximal urban density (plot ratio) for net ZEBs in the chosen cities}

It can be calculated up to which urban density (plot ratio), net ZEB is possible for the different locations. The prerequisite that the energy demand must be covered by renewable energies gained on site sets two limits:

- The yearly harvest of a PV module system on the building's roof can cover the electricity demand (mechanical ventilation, artificial light, heat pump) for a certain number of storeys.

- A borehole heat exchanger system in the ground can deliver a certain amount of power for heating or cooling. With it, the heating and cooling demand of a certain number of storeys can be covered. For the calculation, a building distance of $20 \mathrm{~m}$ is assumed, which corresponds to a typical and realistic street width. From this assumption and the size of the estate, the size of the geothermal system can be determined. In case it is insufficient and there is a reserve in the PV electricity production, standard chillers (COP $=1.5$ ) are further assumed until the PV system is exhausted.

The dominant of the two criteria decides about the maximal number of storeys. The resulting urban density can be finally calculated. Figure 4 shows the results. 


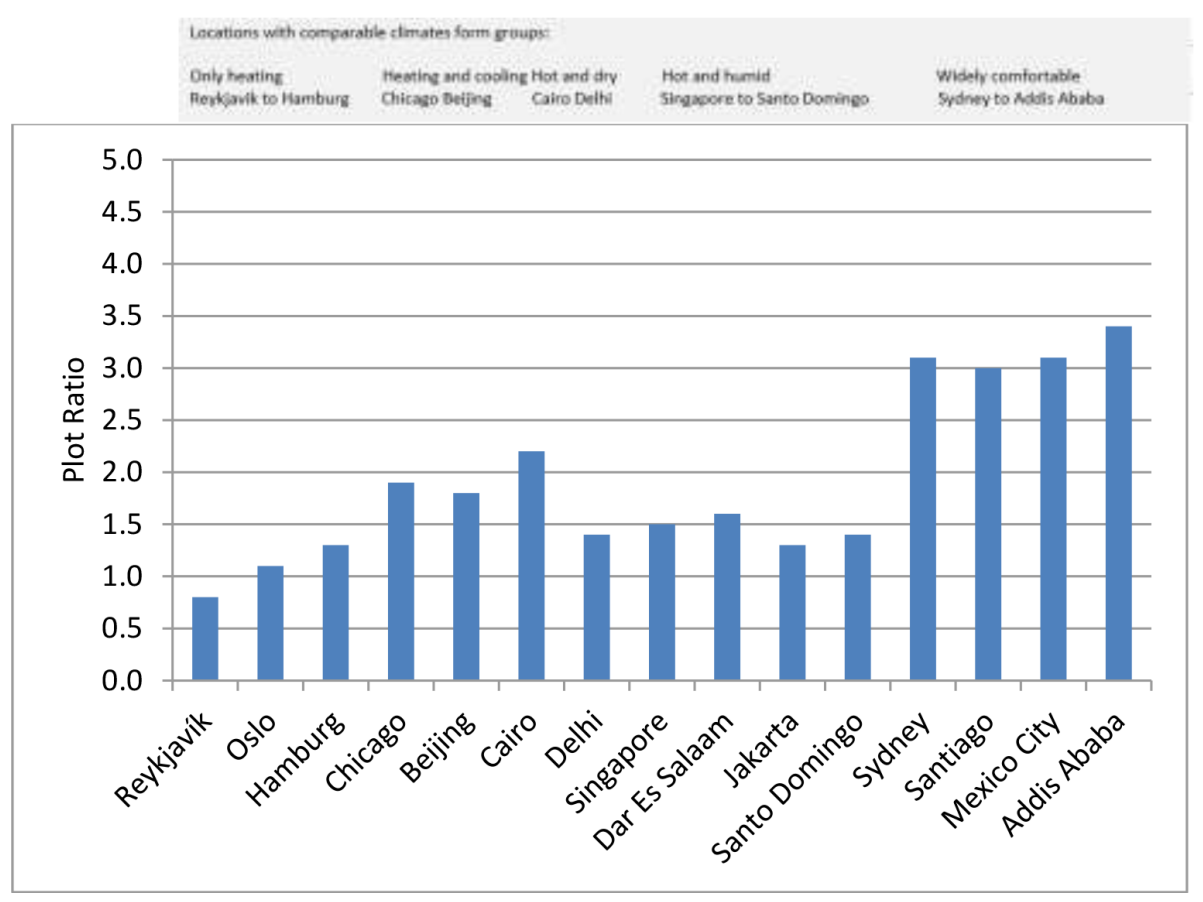

Figure 4: Maximal urban density (plot ratio) for net ZEB for the chosen cities.

From Fig. 4, it is noticeable that Reykjavik has the lowest plot ratio 0.8 which reflects unfavourable conditions prevailing in the city: a strong winter causing high heating demand, extended times of darkness causing demand for artificial light and together with the mechanical ventilation leading to high electricity demand - on the other hand, it has the lowest solar radiation and the sun position hardly above the horizon that results in a very low harvest with PV modules on the roof.

The highest possible plot ratio of 3.4 is shown by Addis Ababa which has a moderate climate. A moderate climate requires nearly no thermal energy for heating or cooling, daylight in the whole time of usage, no mechanical ventilation leading to a minimal electricity demand that can easily be covered with the PV system on the roof receiving a high amount of solar radiation. It is noted that the locations with similar climates show also similar results here. Or, vice versa, the local climatic conditions determine the chances to reach both, net ZEB and a higher urban density.

4.3 Need of compensating land for ZEB_CM for selected urban densities (plot ratio) for the chosen cities

If the planned urban density exceeds the limit for net ZEB, supplementary compensating land for renewable energy production is necessary. That land must be located outside the city (or on the sea in case of offshore wind turbines). It must be evaluated precisely if and where that is available. Competition between energy and food production must be avoided. On the other hand, a city with a high urban density avoids urban sprawl and saves land; a compact city has short distances and saves energy for transportation. The best combination for each location must be found by balancing all impacts. 
The need for compensating land is determined for the urban densities (plot ratio) of two, three and four. It is known from section 4.2 that several cities reach such a high plot ratio partly already without compensating measures but a plot ratio of 4 requires compensating land for all. The results present the total land use, estate plus compensating land.

\subsubsection{Compensating measures wood pellets (heat) and horizontal PV (power)}

The most common systems to produce renewable energies are selected:

- For thermal energy heating, wood pellets out of renewable forestry to be transported and burned on site.

- For electricity, PV modules mounted horizontally.

Logically, for all locations, the land use for the estate decreases with increasing plot ratio. But for high plot ratios, a higher amount of compensating land is necessary, causing an increasing or decreasing total land use (Fig. 5). For the locations without significant heating demand, the total land use decreases with increasing plot ratio (Cairo to Addis Ababa).

But for other locations (Reykjavik to Beijing), the compensating land and with it the total land use increase remarkably with increasing plot ratio. The reason is that these locations have a heating demand that increases with increasing plot ratio and less of this demand is covered with the geothermal system on site. In comparison to a geothermal system, the efficiency of renewable forestry was recognized as very low (see section 3.2.3). Therefore, more energy for heating must be produced on compensating land, so higher the need of land for

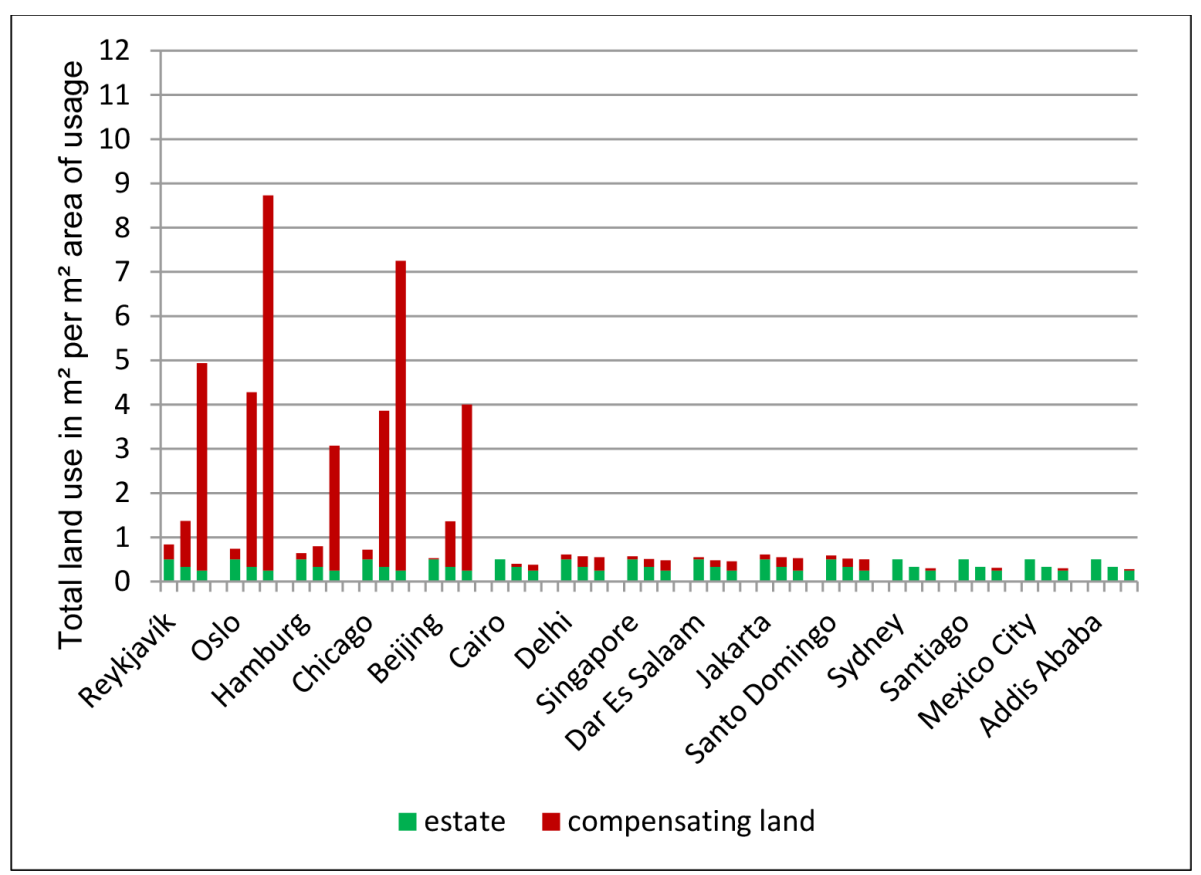

Figure 5: Total land use (estate plus compensating land) in $\mathrm{m}^{2}$ per $\mathrm{m}^{2}$ area of usage with compensating measures wood pellets and PV. The three columns for each city correspond to an urban density (plot ratio) of 2 (left), 3 (middle) and 4 (right). 


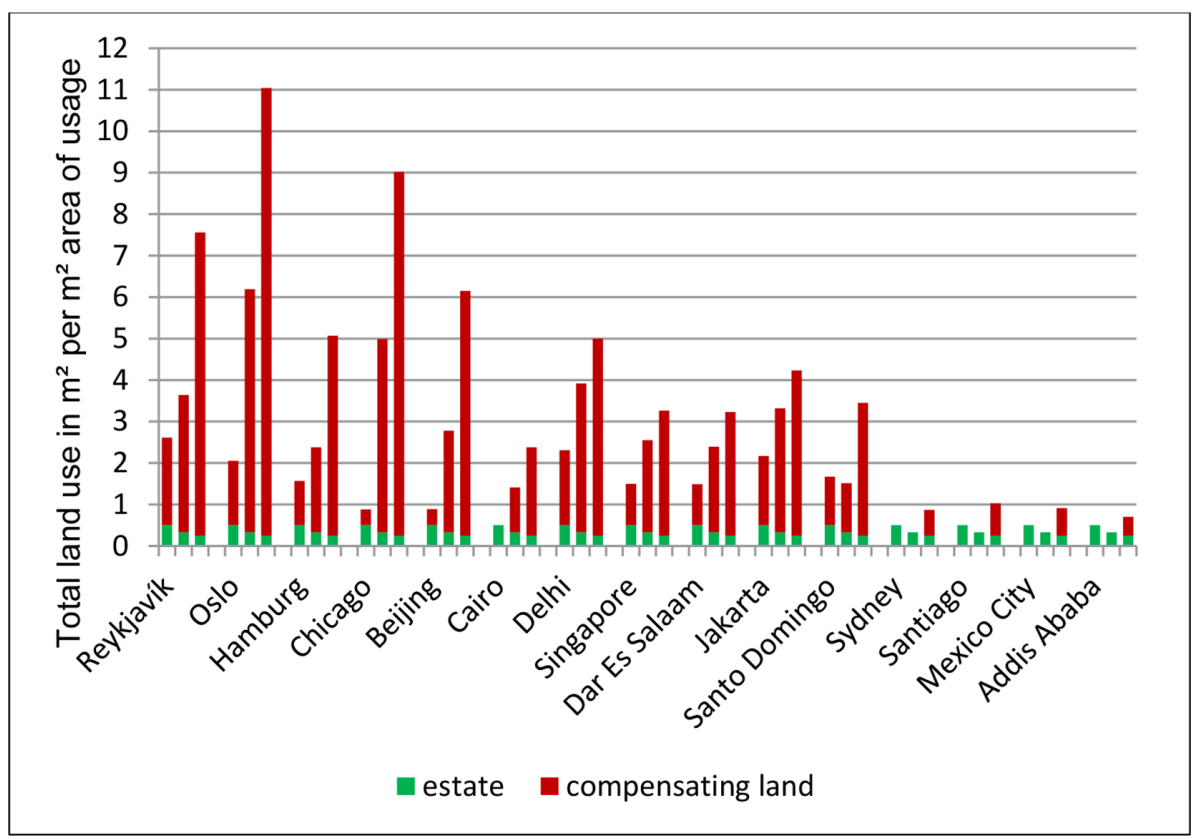

Figure 6: Total land use (estate plus compensating land) in $\mathrm{m}^{2}$ per $\mathrm{m}^{2}$ area of usage with compensating measures wood pellets and wind turbines onshore. The three columns for each city correspond to an urban density (plot ratio) of 2 (left), 3 (middle) and 4 (right).

renewable forestry. That effect is absolutely dominating. It must be decided for each location if such an amount of forest would be available or not.

This paper gives information to the theoretical need of compensating land. Further, it might be important if and how much compensating land would be available in practice, for certainly there are differences between Reykjavik, say, and Singapore.

\subsubsection{Compensating measures wood pellets (heat) and wind onshore (power)}

While there is no alternative to the production of wood pellets for heating, the impact of wind turbines onshore instead of PV modules can be investigated.

Now there is a clear effect that the total land use increases with increasing plot ratio for all chosen cities (Fig. 6). The reason is that PV modules with a higher energy density are replaced by wind turbines with a lower one. On the other hand, as already mentioned, multifunctional land use like agriculture is possible where wind turbines onshore are placed.

Only for locations with moderate climate and (nearly) no demand for heating as well as power, this increase is moderate and could be tolerated (Sydney to Addis Ababa).

\section{CONCLUSIONS}

A city is by definition an organism where a lot of functions are concentrated on a small space. It is not self-sufficient; energy, water, food, goods, etc. must be delivered to the city; waste, products, knowledge, culture, etc. are delivered backward to the surrounding. To find the best sustainable solution, the city and its surrounding must be regarded together. Certainly, the 
city should contribute towards sustainability to unburden its harmful effects and resource depletion in the surroundings and thus the environment. Sufficiency should be the leading principle: reduction of demand by saving energy, material, water, etc. The efficiency of renewable systems to cover the remaining demand is the second principle.

Higher urban densities are of advantage: no urban sprawl, a good precondition for public transportation, bike distances.

Depending on the local climatic conditions and the chosen renewable energy systems, the land use for compensating measures can increase rapidly with increasing plot ratio, with negative impacts.

The best strategy is now as before to cover the maximum energy demand in the city. Geothermal systems have the highest energy densities for heat and cold and PV modules for electricity. Both systems should be used in all possible conditions. The best place for PV modules is the building's roofs in the city.

If compensating measures are necessary, it is important to regard the energy density of the different systems and to choose an option that does not cause extended land sprawl. But the priority is to reduce the energy demand of the buildings which means designing the buildings optimized to the climate and adaptive.

The variant international style building with renewable energy systems is not the real sustainable solution; it would lead to further exhaustion of the resources of our planet. Thus, the architecture of the 21 st century is different, and it is climate adaptive.

\section{REFERENCES}

[1] Towards a zero-emissions, efficient and resilient buildings and construction sector; Global status report for building and construction, 2019. IEA and UN environment programme. https://www.worldgbc.org/sites/default/files/2019\%20Global\%20Status\%20 Report\%20for\%20Buildings\%20and\%20Construction.pdf, accessed on: 21 December 2020.

[2] Energy efficiency for buildings, UNEP. http://www.studiocollantin.eu/pdf/UNEP\%20 Info\%20sheet\%20-\%20EE\%20Buildings.pdf, accessed on: 21 December 2020.

[3] Towards a zero-emission, efficient, and resilient buildings and construction sector; Global status report, 2017. IEA and UN environment programme. https://www. worldgbc.org/sites/default/files/UNEP\%20188_GABC_en\%20\%28web\%29.pdf, accessed on: 21 December 2020.

[4] MacKay, D., Sustainable energy - without the hot air, 2008. http://www.withouthotair. com/, accessed on: 21 December 2020.

[5] HafenCity University Hamburg, Research group REAP, Dietrich, U. Primero software (version 2.0, 2018). www.primerosoftware.de, accessed on: 21 December 2020.

[6] Climate Consultant software (version 6.0, 2018). http://www.energy-design-tools.aud. ucla.edu/climate-consultant/request-climate-consultant.php, accessed on: 21 December 2020. 